Research paper

\title{
A small spacecraft to probe the interior of the Jovian moon Europa: Europa Tomography Probe (ETP) system design
}

\author{
Virginia Notaro, Mauro Di Benedetto, Guido Colasurdo, Daniele Durante, Paolo Gaudenzi, \\ Luigi Imperi, Mirco J. Mariani, Armando Marotta, Gianluca Palermo, Luciano Pollice, \\ Paolo Racioppa, Alessandro Zavoli, Luciano Iess*
}

Department of Mechanical and Aerospace Engineering, Sapienza University of Rome, Via Eudossiana 18, 00184 Rome, Italy

\section{A R T I C L E I N F O}

\section{Keywords:}

Europa: inter-satellite link

System engineering

Geophysics

\begin{abstract}
A B S T R A C T
We propose the system design of a small orbiter of the Jovian moon Europa (Europa Tomography Probe, ETP in short) aimed at unveiling its interior structure. ETP is conceived as a piggyback probe of a larger spacecraft to the Jovian system (such as Europa Clipper). Its payload comprises only a magnetometer and transponder. The former will be used to measure the time-varying, induction magnetic field of the moon at different orbital and rotational frequencies, a measurement inaccessible to a flyby spacecraft. An Inter-Satellite Link (ISL) between ETP and the main spacecraft, enabled by the on-board transponder, will be used to accurately determine Europa's gravity field, rotational state and tidal deformation. By combining magnetic and gravity field measurements, ETP could characterize the interior structure of Europa with an accuracy only attainable by a lowaltitude orbiter, thus constraining the thickness and conductivity of the subsurface ocean. Following the announcement that a 250-300-kg mass allowance was available on the upcoming NASA Europa Clipper spacecraft, we propose ETP's mission and spacecraft design assuming Clipper's nominal trajectory as a baseline. The concept can be adapted to a class of missions to the Jovian and Saturnian systems based upon a mother-daughter spacecraft system. The spacecraft design has been pursued under the philosophy of determining the minimum required total mass and volume that allows to meet the scientific requirements, rather than finding out what science return could be obtained with pre-assigned system constraints. Since ETP shall autonomously reach its final orbit, the propulsion system has been one of the primary focuses. The radiation analysis has also been essential because the shielding structure affects both the mission duration and the mass budget. We show that the ETP concept could be, indeed, technically feasible with an overall system mass budget just above $250 \mathrm{~kg}$, thus providing a valuable yet affordable augmentation to a larger flyby mission to Europa.
\end{abstract}

\section{Introduction}

The Jovian moon Europa is a celestial body of primary interest for planetary scientists. The likely existence of a global subsurface ocean, provided by measurements carried out during the Galileo mission, makes Europa one of the most promising environments in the Solar System to sustain extra-terrestrial habitability conditions. During its scientific operations, the Galileo magnetometer sensed unique perturbations of the Jovian magnetic field near Europa, compatible with the existence of a global subsurface ocean with an Earth-like salinity [1,2]. Galileo's gravity field measurements suggest that Europa has a differentiated structure, where a rocky interior is surrounded by an 80 to $170 \mathrm{~km}$-thick ice-water layer [3], although current estimates indicate that the icy shell can be much thinner. The thickness of the outer icy layer and the depth of the ocean remain essentially unknown. Optical data, which provided a detailed map of the ice-covered surface, are also compatible with the global picture of an icy surface floating on a subsurface ocean. The presence of an ocean, probably sustained by the permanent heat source given by tidal dissipation, implies that Europa could potentially host life [4]. The determination of the ice-water layer's characteristics is a primary objective of NASA's 2013-2022 Decadal Survey [5].

The most scientifically rewarding strategy to determine Europa's interior structure consists of deploying a low-altitude orbiter capable of measuring the time-dependent variations in the gravity and magnetic fields of the moon [6,7]. Although several mission concepts have been studied in the past years [8,9], the orbiter option has always been deemed unfeasible because of excessive cost and mass, mostly due to

\footnotetext{
* Corresponding author.

E-mail address: luciano.iess@uniroma1.it (L. Iess).
} 


\author{
Acronyms/abbreviations \\ AOCS Attitude and Orbit Control System \\ BOL Beginning Of Life \\ CBE Current Best Estimate \\ COTS Commercial Off-The-Shelf \\ EGA Earth Gravity Assist \\ EOI Europa Orbit Insertion \\ EOL End Of Life \\ EPS Electric Power System \\ ETP Europa Tomography Probe \\ FOG Fiber optic Gyroscope \\ HGA High Gain Antenna \\ ISL Inter-Satellite Link \\ LGA Low Gain Antenna \\ MEL Master Equipment List \\ MGAl Mass Growth Allowance \\ MGAn Medium Gain Antenna
}

$\begin{array}{ll}\text { MIPS } & \text { Million Instructions Per Second } \\ \text { MLI } & \text { Multi-Layer Insulation } \\ \text { MMH } & \text { Monomethyl Hydrazine } \\ \text { NTO } & \text { Nitrogen Tetroxide } \\ \text { OBDH } & \text { On-Board Data Handling } \\ \text { PCDU } & \text { Power Control and Distribution Unit } \\ \text { RAAN } & \text { Right Ascension of the Ascending Node } \\ \text { RCT } & \text { Roll Control Thrusters } \\ \text { RW } & \text { Reaction Wheel } \\ \text { SLS } & \text { Space Launch System } \\ \text { SRAM } & \text { Static Random Access Memory } \\ \text { SS } & \text { Sun Sensor } \\ \text { ST } & \text { Star Tracker } \\ \text { TCS } & \text { Thermal Control System } \\ \text { TID } & \text { Total Ionizing Dose } \\ \text { TRL } & \text { Technology Readiness Level } \\ \text { TT\&C } & \text { Telemetry Tracking and Command } \\ \text { USO } & \text { Ultra-Stable Oscillator }\end{array}$

the harsh radiation environment around the moon. Currently, two planetary science missions are planned to flyby Europa, ESA's Jupiter Icy worlds Explorer (JUICE), and NASA's Europa Clipper. The former will be launched in 2022 and will perform several flybys of Callisto (plus two of Europa), until entering into orbit around Ganymede. The NASA spacecraft, instead, will be launched in the 2020s and will perform 45 flybys of Europa for a total mission duration of three years $[10,11]$. The nominal trajectories of both spacecraft ensure that they will remain mostly outside the Jovian radiation belts, thus waiving the need of a large shielding mass. However, the Cassini-like multiple flyby approach is not favorable to constrain the thickness and conductivity of Europa's subsurface ocean.

We present an innovative mission concept consisting of a small spacecraft that would orbit Europa to accurately characterize its interior structure. The Europa Tomography Probe is proposed as an augmentation to Europa Clipper following NASA's announcement in 2015 that a $250-300-\mathrm{kg}$ allowance was available for an additional flight element on the main spacecraft. ETP hosts a payload made up only of a magnetometer and a transponder, and will autonomously acquire and maintain a 250-km polar orbit around Europa for up to 6 months. The magnetometer will continuously measure the variable magnetic field of Europa at different frequencies. The transponder will be used to establish periodic Inter-Satellite Link (ISL) windows with the mother spacecraft to perform Doppler measurements and determine the moon's gravity field. The combination of these two measurements, inaccessible to a flyby spacecraft such as JUICE or Europa Clipper, could allow solving for the thickness and conductivity of Europa's subsurface ocean. ETP will also provide a much-improved positioning of Europa Clipper with respect to Europa, therefore enabling a measure of the physical tides of the moon using the powerful radar REASON. The combined measurement of the gravitational and physical tides will provide also the thickness of the outer icy shell [7]. In a companion paper, Di Benedetto et al. [12] present further details on the science motivation behind ETP and the results attainable from the gravity science experiment, as well as the overall science enhancement of the Clipper mission.

This paper is focused on the system architecture of the probe as derived from the scientific requirements and the mass constraint. It is structured as follows. Section 2 describes the spacecraft design method. Section 3 lists the scientific requirements and Section 4 describes the reference mission profile. Section 5 , which is the core of this paper, describes the spacecraft design and its architectural configuration. Section 6 presents the final mass budget, providing considerations about the flexibility of the proposed design for other main spacecraft configurations and mission profiles.

\section{Spacecraft design method}

The feasibility study presented in this paper was motivated by NASA's 2015 announcement that a 250-kg mass allowance was available on the forthcoming Europa Clipper spacecraft. This opportunity, open to non-US contributions, triggered considerable interest especially in Europe, where the planetary science community and industry outlined possible uses of the available mass. Although Clipper hosts a complete suite of scientific instruments dedicated to the investigation of Europa's outer shell and plasma environment, the nominal mission profile is not optimal for investigating the interior structure of the moon. As previously mentioned, a low-altitude orbiter is possibly the most appropriate option to probe the subsurface ocean of Europa. Previous studies of orbiters proposed as an additional flight element for Europa Clipper included complex scientific instrumentation, such as plasma sensors and optical instruments that would study the presence of chemicals on the surface of the moon and the composition of gas plumes observed by NASA's Hubble Space Telescope in the southern regions of Europa [13]. Thus, the resulting mass budgets exceeded the 250-kg limit by several hundred kilos.

Following NASA's announcement, instead, we proposed a small probe with a minimal payload (just a magnetometer and a transponder) complementing and enhancing the science return of a large flyby mission (Europa Clipper) while still meeting the strict mass constraint. The use of small spacecraft (weighing less than $500 \mathrm{~kg}$ ) for deep space exploration represents an emerging concept among space agencies and research institutions as it is a viable design choice to augment larger missions with a limited added mass and cost increase [14]. We assessed the feasibility of the Europa Tomography Probe concept by formulating a complete set of scientific objectives (Section 3) and assessing the added scientific return as opposed to that provided by Clipper alone; the results are described in Ref. [12]. Then, as described below, we addressed the technical feasibility of ETP, especially regarding the total mass budget. The preliminary design of the spacecraft and the definition of each subsystem have been carried out and iterated several times as per the consolidated approaches reported in the reference literature [15-20]. When defining ETP's mass budget, we focused on the state-ofthe-art small satellite technology [21]. As small satellite components are mostly tailored towards near-Earth applications, their radiation hardness was a crucial driver during component selection and led to several system trade-offs between mass and shielding thickness. Since this study can be classified as Phase 0 or Mission Concept Study (as per ESA/NASA classification) an appropriate mass margin definition has been a primary concern. According to NASA/JPL flight design principles and AIAA's guideline on mass properties control for space flight 
systems [22,23], the Current Best Estimate (CBE) mass of a component or subsystem is the basic value of the mass measured or computed at the latest design status. Then, a $5-15 \%$ Mass Growth Allowance (MGAl) is added to the CBE to take into account the mass increase due to advanced design stages that lead to higher Technology Readiness Level (TRL). A final 5-35\% mass margin on top of the predicted mass (CBE plus MGAl) represents the contingency due to unpredictable effects that could cause an increase in mass (for example, changes in the reference trajectory and thus in the required propellant mass). The total margin (composed of MGAl and mass margin) can range from 25 to $40 \%$ depending on the mission status and criticalities in the design [24]. The actual values of the MGAl and mass margin selected for the analysis presented in this paper are based on the following considerations. Early concept phase designs such as the one presented in this paper generally require a mass margin of at least $25 \%$ [22]. Furthermore, although many of the components selected for ETP are TRL $>6$, they are mostly tailored towards Earth-bound applications, so that the MGAl shall take into account the increased radiation shielding required to adapt the already flight-proven design to deep-space missions. Consequently, we varied the MGAl from $5 \%$ to $15 \%$ according to the TRL and radiation hardness of the single components (see Section 6). Regarding the margin on the predicted mass, we assumed the maximum recommended value of $30 \%$ due to the large number of uncertainties on the final spacecraft design, mainly related to the radiation environment [22].

The system and mission design described in the following sections and in Ref. [12] have been carried out by assuming Europa Clipper's nominal trajectory as a reference. However, regardless of whether the additional mass allowance will still be considered, we demonstrate that the concept of a small probe with minimal payload that can augment the scientific return of a larger mission to Europa is feasible. The proposed design can indeed be flexibly adapted to future missions to the Jovian moon.

\section{Mission requirements}

The main goal of ETP is to employ a combination of continuous measurements of Europa's magnetic field and an accurate estimate of the moon's gravity field though the ISL with the main spacecraft to determine independently the depth and conductivity of the subsurface ocean.

\subsection{Scientific requirements}

In order to fulfill this objective, ETP will exploit the magnetic induction sounding technique, that consists of measuring the perturbed Jovian magnetic field at Europa at both synodic $(11.1 \mathrm{~h})$ and orbital $(85 \mathrm{~h})$ periods. When a conducting medium is bathed in a time-varying magnetic field, electrical currents start to flow and generate secondary magnetic fields. By measuring such fields, it will be possible to confirm the presence of a global ocean under Europa's surface and constrain both its thickness and conductivity [25]. ETP's magnetometer shall be able to sense the smallest expected amplitude variation of the magnetic field, $\sim 7 \mathrm{nT}$ at Europa orbital frequency over a background field of $450 \div 500 \mathrm{nT}$ [26]. Moreover, the magnetic measurements shall be continuously performed for at least three months in a low-altitude polar orbit to obtain a complete map of the variable magnetic field. The orbital altitude shall be less than $1000 \mathrm{~km}$ (a $200-250-\mathrm{km}$ orbit would be optimal).

ETP will measure the static and time-varying gravity field of Europa through Doppler measurements carried out with a two-way coherent microwave inter-satellite link that will be performed for about 6 months, corresponding to 9 Europa Clipper's flyby according to the current trajectory [12]. Precise knowledge of Europa's gravity field will shed light on its interior structure, including the depth of the subsurface ocean. In particular, the gravity experiment will focus on determining
Europa's static gravity field to degree and order 30 of the classical spherical harmonic expansion, although higher-order terms could be accessible. The parameters of interest are estimated as part of ETP's orbit reconstruction process. The time-varying, degree- 2 part of the gravity field, induced primarily by the non-negligible eccentricity of the satellite, will be measured at different anomalies along the orbit of Europa to allow estimating the Love number $k_{2}$ with an absolute accuracy lower than 0.0001. $k_{2}$ accounts for variations in Europa's gravity potential (and shape) due to the differential attraction exerted by Jupiter along the eccentric orbit of the moon, and it depends on the presence and characteristics of the ocean. Since ETP acts as a reference point during the Doppler measurements, the use of the ISL can also significantly improve the accuracy of the main spacecraft's trajectory reconstruction. Radial positioning during flybys at the (sub) meter level could enable a very accurate estimation of the $h_{2}{ }^{1}$ Love number, provided that a laser altimeter is foreseen among the mother spacecraft's payload (such as the REASON instrument on Europa Clipper). The reference signal for the ISL will be generated on the mother spacecraft by means of an Ultra-Stable Oscillator (USO). Doppler measurements will also be produced on-board Clipper.

The combined measurement of the Love numbers and magnetic field intensity will allow constraining the icy shell thickness with an accuracy impossible to achieve with a flyby spacecraft such as Clipper. In particular, it can be shown that an accuracy of $\pm 30 \mathrm{~km}$ is attainable, assuming that the $h_{2}$ Love number can be determined to $\sim 0.05$ and considering a 6-month mission lifetime [12].

\subsection{Spacecraft requirements}

The main system requirement specifies that the final mass of the probe shall fit within the $250-300 \mathrm{~kg}$ range. Furthermore, the magnetic field measurements require knowledge of the spacecraft attitude with respect to the inertial frame with an accuracy of $0.05^{\circ}$. The harsh environment at Europa imposes additional operational requirements. The power, thermal, radiation shielding and propulsion subsystems shall indeed be designed to sustain efficient payload operations throughout the 6-month mission.

\subsection{Payload description}

The magnetometer is composed of three sensors that measure the amplitude and orientation of the magnetic field vector with a frequency of $0.1 \mathrm{~Hz}$. The measurement range is $\pm 1000 \mathrm{nT}$ and the accuracy is 1-2 nT.

The transponder is the core of the telecommunication subsystem but since it also supports radio science measurements, it can be considered as part of the payload. This component will be used to establish a twoway coherent X-band ISL with the mother probe, providing highly accurate Doppler measurements during the ISL windows. The fractional frequency stability of the USO used to generate the reference signal on the main spacecraft is expected to be to the level of a few $10^{-13}$ at 1-1000 s integration times, according to the current state-of-art technology [27]. This could represent a serious limiting factor to the accuracy of Doppler measurements. However, thanks to the short roundtrip light time during the ISL due to the short distance between the two spacecraft $(<1 \mathrm{~s})$, the on-board clock noise is largely suppressed, and the attainable measurement accuracy is comparable to that granted by Ka-band tracking from ground [28].

Both the magnetometer electronics and the transponder require shielding from radiation and should thus be placed in a radiation vault (see Section 5), while the magnetometer sensors should be located as far as possible from the spacecraft bus. Since ETP is a solar-powered

\footnotetext{
${ }^{1}$ The $h_{2}$ Love number represents the tidal deformation of Europa in the radial direction.
} 
probe, the panels are most appropriate to host the magnetometer sensors, although a boom could also be considered in the spacecraft design.

\section{Mission overview}

Table 1 presents a list of ETP's mission parameters, referenced to the nominal Europa Clipper trajectory. Each item in the table is detailed in the following paragraphs.

\subsection{Launch}

Clipper will host ETP for the launch and cruise phases. The nominal mission profile envisions a launch in June 2022 with the Space Launch System (SLS). The launcher can bring Clipper and the secondary probe to Jupiter in 2.7 years using a direct trajectory. Alternatively, the Europa Clipper team is considering a Delta IV Heavy launcher. The vehicle can insert the two spacecraft in a $\Delta V$-Earth Gravity Assist (EGA) trajectory that would last 4.7 years.

\subsection{Cruise phase}

During the cruise phase, the instruments will be switched off; however, vital subsystems such as the on-board computer and the heaters shall be operative. The basic functions of the probe will be powered by Clipper through an electrical interface. Furthermore, a data cable will deliver any possible change in the pre-insertion trajectory.

ETP will be physically connected to the main spacecraft by mechanical interfaces that can transfer the launch loads to the primary structure. The cylindrical structure of the probe, shown in Section 5, is most efficient when subject to axial loads. Clipper's current structure is cylindrical as well, with the High Gain Antenna (HGA) placed on top [29]. Therefore, the most reasonable volume allowance for an additional probe would be on the side, meaning that ETP's structure would have to sustain flexural loads during launch. Given the intrinsic uncertainty on the main spacecraft's configuration, the mechanical interfaces have not been analyzed in this study. However, the large mass margin accounted for the structural subsystem should allow for future changes without severe impacts on the final mass budget, as well as providing flexibility to adapt the ETP concept for future large planetary science missions to the Jovian moon.

\subsection{Pre-insertion and Europa orbit insertion (EOI)}

In the early phase of this study, a direct EOI maneuver requiring an average $\Delta \mathrm{V}$ of at least $3 \mathrm{~km} / \mathrm{s}$ was considered. Since the system mass budget resulting from this mission baseline was well over $500 \mathrm{~kg}$ (thus against the overall idea of a small probe), we referenced the propulsion system design to the pre-insertion flyby trajectory designed specifically for ETP in [30]. Thus, after being released by the main spacecraft, ETP will perform 8 flybys of Europa and 1 of Ganymede until achieving a 6:5 resonant orbit with Europa. The pre-insertion phase will last for $\sim 130$ days; at the end of this period, ETP will perform a 25-min burn to achieve its final orbit. The overall $\Delta \mathrm{V}$ required for the pre-insertion phase and the EOI is about $954 \mathrm{~m} / \mathrm{s}$, which includes an additional $8 \mathrm{~m} / \mathrm{s}$ contingency $\Delta \mathrm{V}$ per flyby to account for trajectory dispersion, following the approach used in the trajectory design for the JUICE mission [31]. Although the additional period spent in the Jovian system will increase the total radiation dose, the pre-injection phase is spent relatively away from Europa and from Jupiter's radiation belts, with consistent benefits on the final mass budget.

\subsection{Orbital phase}

The orbital altitude listed in Table 1 is optimal for the magnetic field measurements. The orbital inclination value has been computed to obtain a dawn-dusk Sun-Synchronous orbit, beneficial for the solar- powered probe. Thus, ETP will not experience regular orbital eclipses, but only short occultation periods (maximum $2.9 \mathrm{~h}$, every $85 \mathrm{~h}$ ) when Jupiter crosses the Europa-Sun line.

ETP's orbit belongs to the unstable class of low-altitude polar orbits around planetary satellites, characterized by a short natural lifetime (on the order of days) due to the third-body perturbation caused by the primary planet. The disturbing force produces an exponential increase in eccentricity while leaving the orbital energy constant, eventually leading to an impact with the central planet. These orbits are, however, ideal for many scientific purposes, and therefore their stability has been extensively studied in the past. Several papers describe methods to increase the natural orbit lifetime, for example by optimizing the initial argument of perigee $\omega_{0}[32,33]$. The evolution of ETP's eccentricity, shown in Fig. 1, has been obtained for a reference case by numerically integrating the three-body problem equations, following the approach in Ref. [32]. The Sun-Synchronous condition used to obtain $i_{0}$ has been modified to include the effects of the third-body perturbation (see Equation 13 of [34]). $\omega_{o}$ has been selected through a trial-and-error process focused on obtaining the longest orbit lifetime. Without active maintenance, ETP would collide with Europa in less than two months. Hence, periodic orbit maintenance is mandatory for the mission. The optimal one-burn maneuver frequency that allows to minimize the total propellant, computed through a parametric optimization, is 11 days. The reference orbital parameters selected to produce Fig. 1 are optimized for the mission's scientific objectives. Injection errors and uncertainties may cause discrepancies from this solution and, although a complete error analysis is not the aim of this paper, these effects can reasonably be included in the mass margin on the propellant.

\subsection{End of mission}

At the end of the nominal mission, the orbit will be left to drift naturally. This End-Of-Life (EOL) strategy shall be planned with particular attention to comply with the planetary protection guidelines established by the Committee on Space Research. ETP is indeed a Class-IV spacecraft as it will orbit and impact a body of scientific interest for the possible presence of life. Hence, during the realization and testing of the probe, appropriate countermeasures such as those listed in Table 1.1 of [35] must be implemented. As an example, a Class-IV spacecraft shall be partially or totally sterilized in a clean room, and the bioburden (i.e., the number of non-sterilized bacteria) shall be continuously monitored.

\section{Spacecraft design}

Table 2 presents an overview of ETP's nominal configuration and a summary of the main system characteristics. The spacecraft is composed of a bus module, which hosts the avionics and the subsystem components, and of a propulsion module that contains the propellant and thrusters. The probe can communicate with the main spacecraft either through a conical horn Medium Gain Antenna (MGAn) or via two Low Gain Antennas (LGA). A central cylinder structure, aided by four

Table 1

ETP reference mission profile.

\begin{tabular}{ll}
\hline Parameter & Description \\
\hline Mission objective & Determine Europa's interior structure \\
Estimated launch date & June, 2022 \\
Cruise phase duration (yrs) & 2.7 (Direct)/4.7 ( $\Delta$ V-EGA) \\
Pre-insertion duration (days) & 131 \\
Scientific mission duration (months) & 6 \\
Science orbit & Sun-Synchronous (dawn-dusk) \\
Orbital altitude (km) & 250 \\
Eccentricity & $<0.00001$ \\
Inclination $\left(^{\circ}\right)$ & 91.18 \\
Max. eclipse duration (hrs) & 2.9 every Europa period \\
EOL strategy & Natural orbit decay \\
\end{tabular}




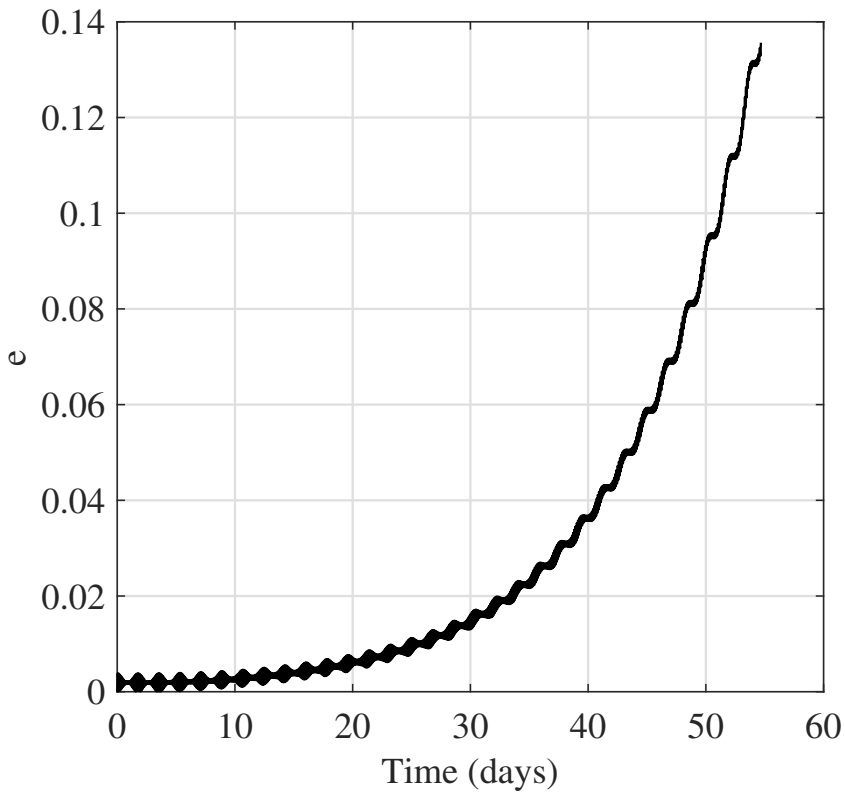

Fig. 1. Natural eccentricity evolution until impact with Europa. The initial conditions on the eccentricity $\left(e_{0}\right)$, the semi-major axis $\left(a_{o}\right)$, the inclination $\left(i_{0}\right)$, the argument of perigee $\left(\omega_{0}\right)$ and the RAAN $\left(\Omega_{0}\right)$ used for this simulation are: $\mathrm{e}_{0}=0.00001, \mathrm{a}_{0}=1810 \mathrm{~km}, \mathrm{i}_{0}=98.184^{\circ}, \omega_{0}=375^{\circ}, \Omega_{o}=0^{\circ}$.

shear panels, provides the primary load path. The structural subsystem includes supporting elements for each component.

The bus view in Fig. 2 contains a detailed overview of the spacecraft subsystems, which will be discussed in the following paragraphs. Moreover, Fig. 7 provides a functional block diagram of the bus.

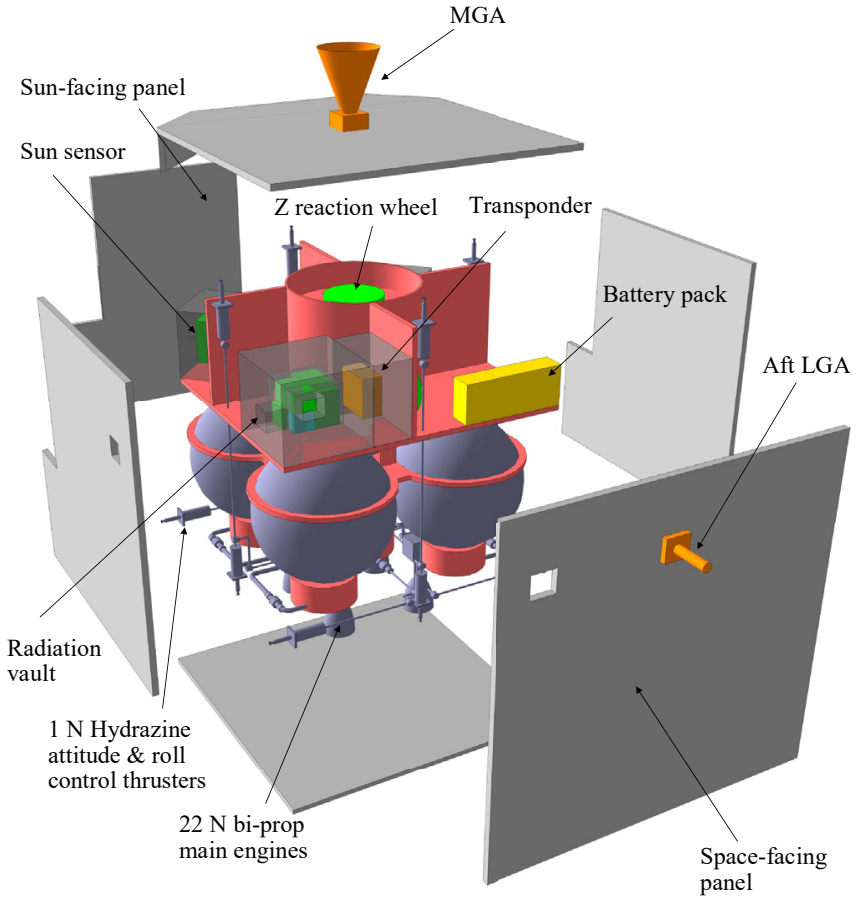

Fig. 2. ETP bus exploded view. Colors in the figure represent different subsystems: structural subsystem (red and grey, outer panels), attitude control system (green), EPS (yellow), TT\&C (orange), propulsion system (violet), OBDH (blue), payload electronics (dark red, not visible). Solar panels have been removed for visual clarity. (For interpretation of the references to colour in this figure legend, the reader is referred to the Web version of this article.)

Table 2

Spacecraft overview.

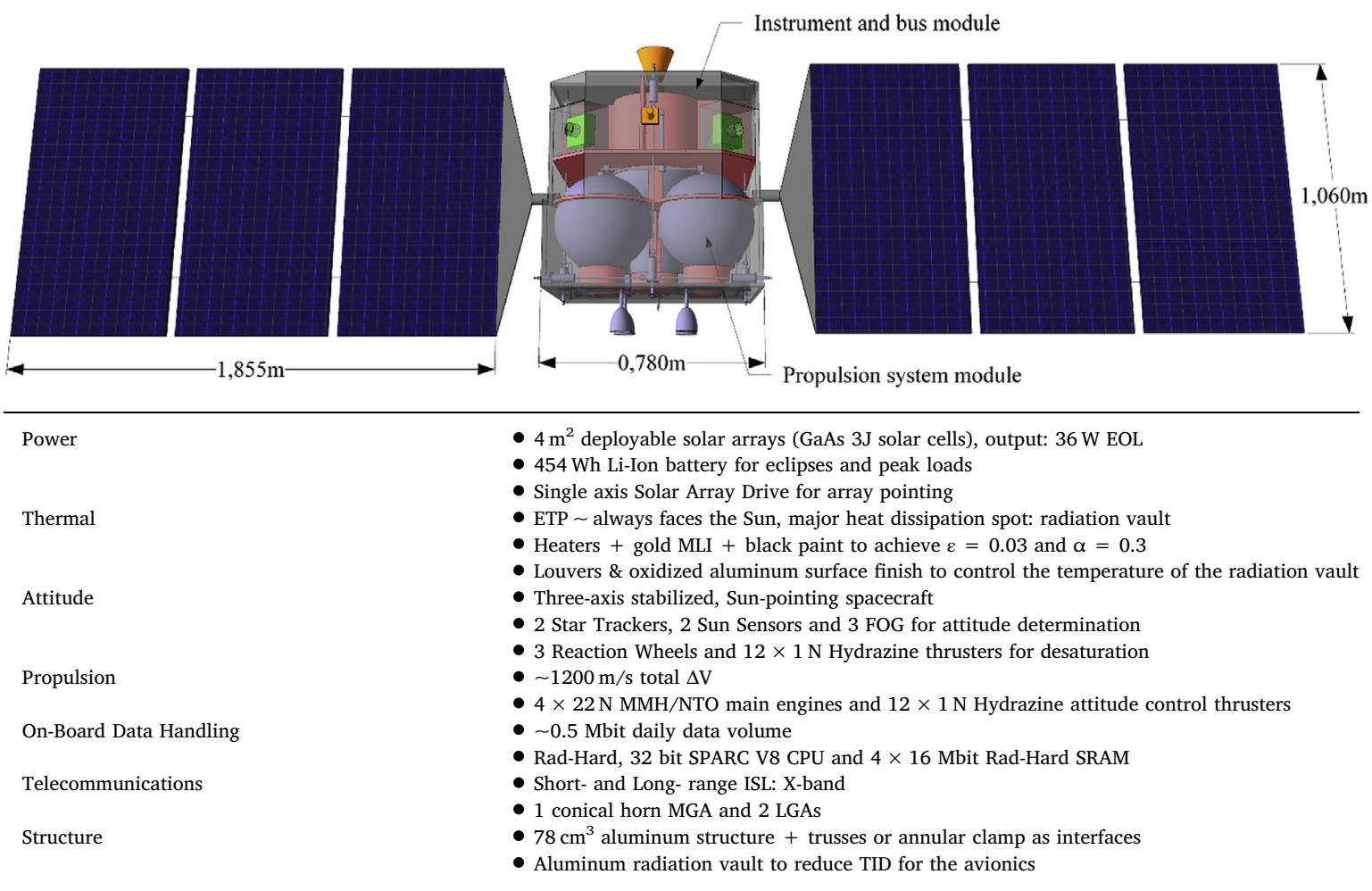




\subsection{Propulsion system}

The propulsion system shall execute the EOI maneuver, regularly maintain the orbit against perturbations, as well as desaturate the reaction wheels. The overall required $\Delta \mathrm{V}$ is $997 \mathrm{~m} / \mathrm{s}$, which includes the pre-insertion corrections $(159 \mathrm{~m} / \mathrm{s})$, the EOI $(716 \mathrm{~m} / \mathrm{s})$, the contingency for trajectory dispersion after the flybys and the overall orbit maintenance $(43 \mathrm{~m} / \mathrm{s})$.

The primary propulsion system comprehends 4, 22-N MMH/NTO thrusters that are fired in pulsed mode to allow thrust vector control, which, in turn, provides two-axis attitude control during the main burn. The third axis (i.e., the thruster's axis of symmetry) is instead controlled by 4 secondary Roll Control Thrusters (RCT), placed at the spacecraft corners. The secondary propulsion system is composed of the 4 RCTs and two redundant sets of 4 thrusters; the 12 thrusters provide threeaxis attitude control for desaturation maneuvers. The attitude control thrusters are based on a monopropellant hydrazine feed system. Fig. 3 shows a schematic of the propulsion system, while the implemented architecture can be visualized in Fig. 2 .

\subsection{Attitude determination and control system}

Although Star Trackers (ST) can satisfy the requirement on the attitude determination accuracy, these sensors are subject to false-star detections in harsh radiation environments. This leads to a large computational cost that can be reduced using the Active Pixel Sensor technology, currently not employed on small satellite sensors. However, small satellite STs represent an advantageous and mass-efficient method to satisfy the mission requirements and, assuming that the technology gap will be filled in the coming years thanks to the increasing interest for small probes for deep-space exploration, two sensors have redundantly been selected as primary attitude determination devices. The attitude determination system includes also two Sun Sensors (SS) (for fine solar array pointing) and 3 Fiber Optic Gyroscopes (FOG). The accuracy of the STs is 6 arcsec around the boresight axis, while that of the SSs is $0.02^{\circ}$ (about 70 arcsec) up to $50 \mathrm{AU}$.

ETP will maintain a Sun-pointing reference attitude to optimize energy production in the low-illumination, low-temperature Jovian environment. Any disturbance to the attitude (mostly gravity gradient torque from Europa and solar radiation pressure torque) is counteracted by 3 Reaction Wheels (RW). To account for variable ISL geometry, a worst-case repointing maneuver has thus been included in the RW design. The angular momentum storage of the selected RWs $(0.5 \mathrm{Nm})$ fits the estimations, with a saturation period of about 30 days; nonetheless, desaturation is still required before every ISL window. For the attitude control system design, we estimated the moments of inertia analytically by reducing the complete spacecraft configuration shown in Fig. 2 to simpler shapes. The algorithm used to obtain the solution has also been run to predict the displacement of the center of mass during the main burn. Results have then been validated by a static analysis performed on a simplified Finite Element mmodel of the spacecraft.

Table 3 lists the operative modes of the probe. The first two modes are critical; during pre-insertion and orbital insertion, the core subsystems shall indeed be functional. In addition, the TT\&C subsystem must be fully operational to allow determining the trajectory of the probe. The magnetometer payload is only active during the orbital modes (3-6). Moreover, in case of a fault, the on-board computer activates the Safe Mode (7) and switches the SSs on to regain a Sunpointing attitude, while waiting for additional commands from the main spacecraft. The radio frequency input/output is consequently transferred from the MGAn to one of the two LGAs.

\subsection{Electrical power system}

Two solar panels with an overall area of $4.2 \mathrm{~m}^{2}$ will power ETP during its mission. The panels are made of triple-junction GaAs solar cells that provide a state-of-the-art efficiency of $29 \%$. Because of the low temperature and solar flux, as well as the high level of radiation, the solar panels degrade quicker than in an Earth-orbiting mission. The degradation factor $L_{d}$ is defined in Equation (1), where $P_{E O L}$ and $P_{B O L}$ are the solar panel specific power outputs at EOL and BOL, respectively:

$L_{d}=\frac{P_{E O L}}{P_{B O L}}$.

We selected $L_{d}=0.87$ to account for the particularly harsh environment at Europa [36]. The total area has been sized with the power consumption data shown in Fig. 4: the arrays shall sustain the average load level in the sunlight orbital modes ( 3 and 4), plus a $25 \%$ margin. Assuming an accurate Sun pointing granted by a one-axis Solar Array Drive motor, the resulting EOL power output is $35.8 \mathrm{~W}$. Furthermore, a Li-Ion battery with an overall energy capacity of $454 \mathrm{Wh}$ has been sized to handle every peak load (like that in mode 2) and power ETP during occultations.

The power regulation and conditioning system is based on a SunRegulated, Direct Energy Transfer bus with a $28 \mathrm{~V}$ reference voltage. Three power lines selected by switches are included in a Power Control and Distribution Unit (PCDU) to satisfy the voltage requirements of each component (Fig. 7).

\subsection{Thermal control system}

The temperature environment has been examined by developing an analytical thermal model with ETP treated as a one-node sphere. We included the following heat sources: solar radiation, albedo and infrared emission from Europa and Jupiter, as well as the spacecraft internally dissipated power. The main dissipation spot inside the probe is the radiation vault: the enclosed electronics consume $15 \mathrm{~W}$, on average,

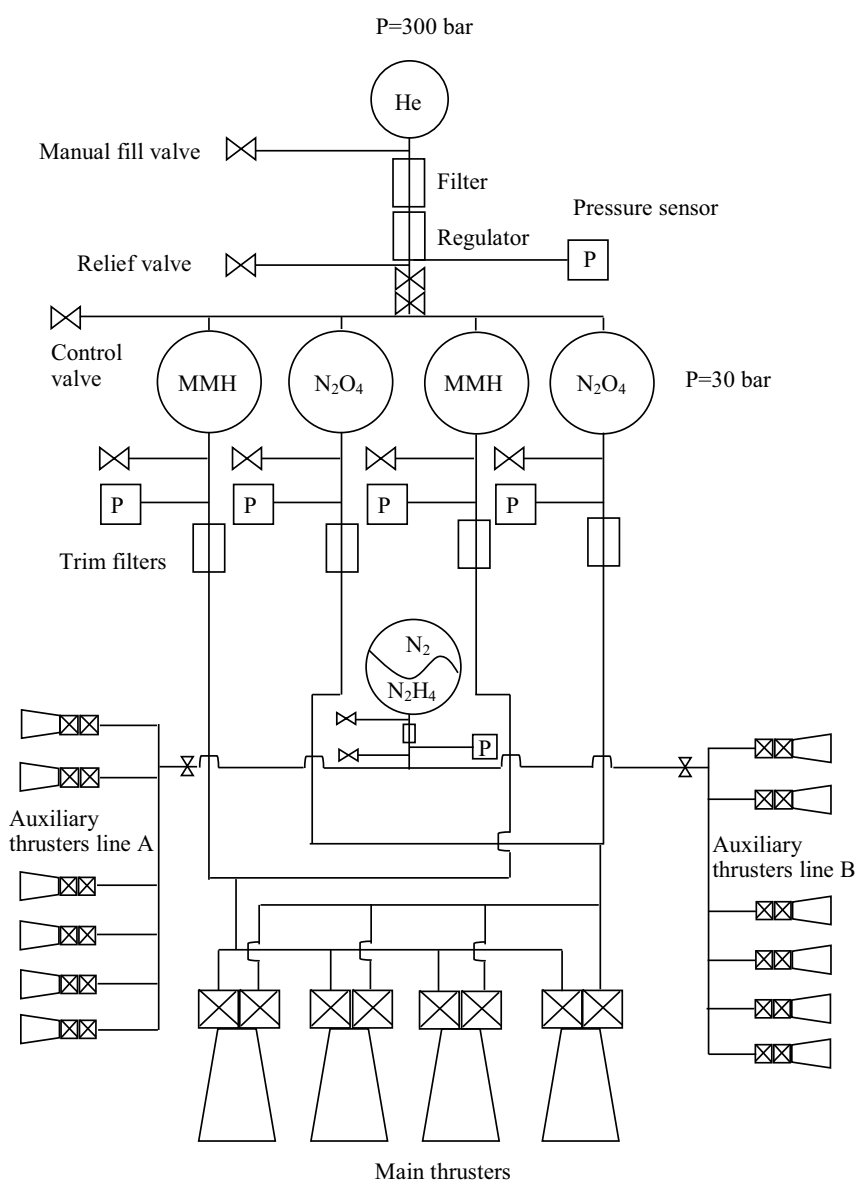

Fig. 3. Propulsion system schematic. 
Table 3

ETP operative modes; the items marked with $\times$ are active in the current mode. Mode legend: 1: Pre-EOI Mode, 2: EOI Mode, 3: Orbit (Sunlight, no ISL) Mode, 4: Orbit (Sunlight, ISL) Mode, 5: Orbit (Eclipse, no ISL) Mode, 6: Orbit (Eclipse, ISL) Mode, 7: Safe Mode.

\begin{tabular}{lcccccc}
\hline Mode & ST & SS & FOG & RW & TT\&C & MAG \\
\hline 1 & $\times$ & $\times$ & $\times$ & & $\times$ & \\
2 & & $\times$ & $\times$ & & & \\
3 & $\times$ & & & $\times$ & & $\times$ \\
4 & $\times$ & & $\times$ & $\times$ & $\times$ & $\times$ \\
5 & $\times$ & & & $\times$ & & $\times$ \\
6 & $\times$ & & $\times$ & $\times$ & $\times$ & $\times$ \\
7 & $\times$ & $\times$ & & & $\times$ & \\
\hline
\end{tabular}

Power consumption (W)

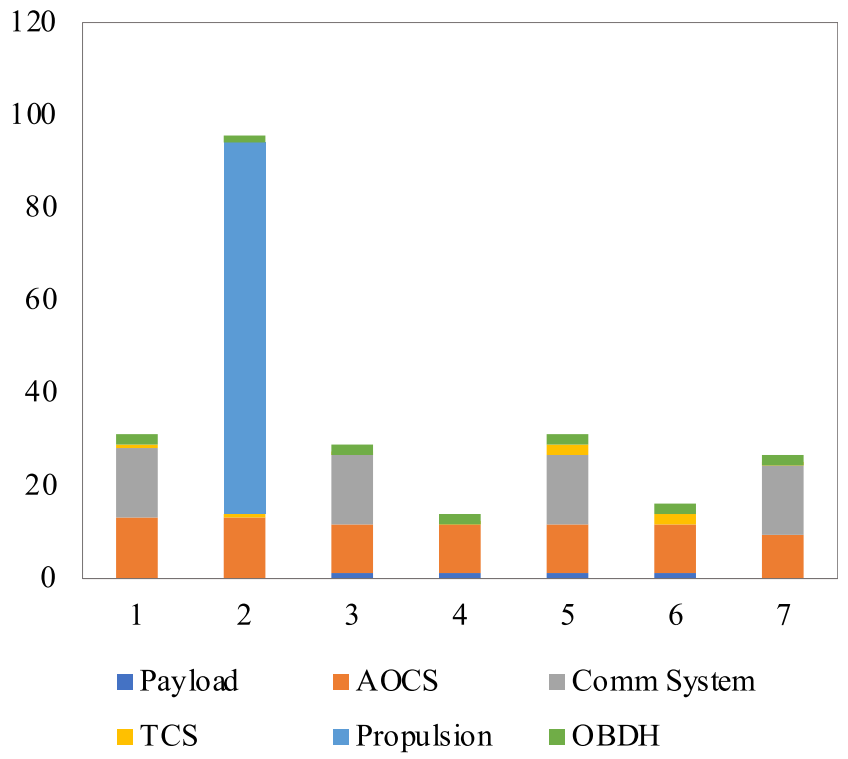

Fig. 4. Power budget for each operative mode defined in Table 3.

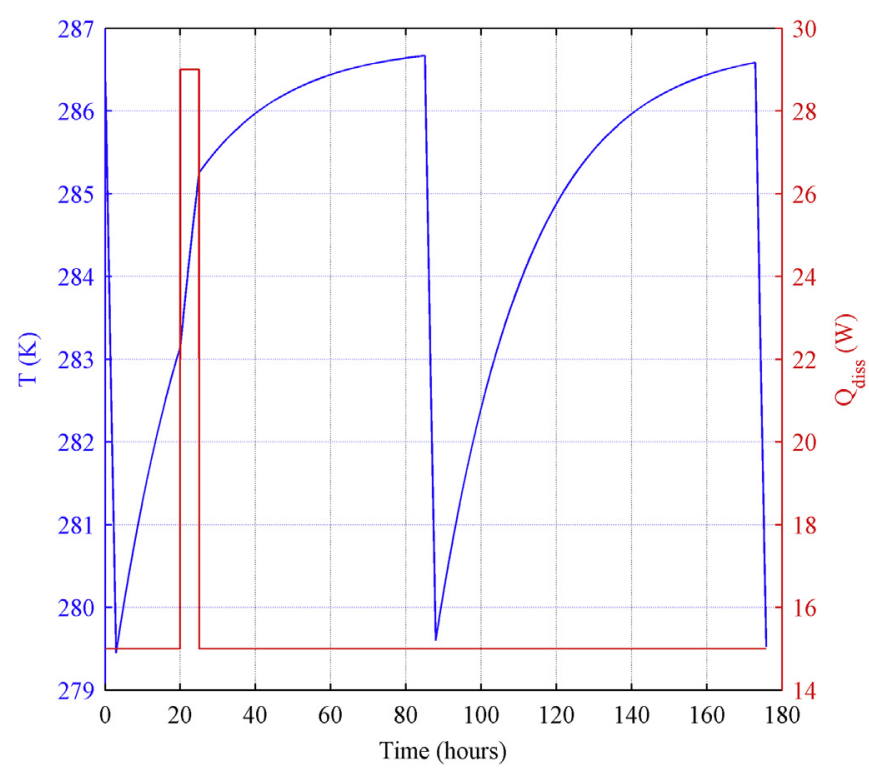

Fig. 5. Spacecraft temperature behavior (blue) and dissipated power (red) over two Europa periods. (For interpretation of the references to colour in this figure legend, the reader is referred to the Web version of this article.) during nominal operations. Since the mean solar input at Jupiter is only $\sim 50 \mathrm{~W} / \mathrm{m}^{2}$, high absorptivity $(\alpha)$ and low emissivity $(\varepsilon)$ coefficients are desirable. A $\alpha / \varepsilon$ ratio equal to 10 has been selected from the equilibrium analysis as it ensures a spacecraft temperature above the stringent requirements of the STs and batteries $(273 \mathrm{~K})$. This is attainable with an appropriate combination of Gold MLI blankets and black paint. However, the thermal control system is not completely passive; localized heaters must indeed be placed along the propulsion feed lines to maintain the propellants within their design temperature range.

We also carried out a transient analysis by integrating the energy evolution equations over two orbital periods of Europa. A 4-h communication window has been included during the first sunlight period, as shown in Fig. 5. Thanks to the large sunlight-over-eclipse time ratio, the temperature of the probe always remains close to the sunlight equilibrium, dropping by a few degrees during occultation periods. The thermal analysis for the solar panels revealed that their sunlight equilibrium temperature is below $150 \mathrm{~K}$ due to the low solar flux. The behavior of the solar cells under these conditions can be predicted using the models developed in Ref. [36].

Another issue for the thermal control system is the temperature control inside the radiation vault. Without countermeasures, temperatures as high as $350 \mathrm{~K}$ can be reached inside the vault during the most power-intensive operative modes. A higher emissivity of the vault, such as that attained with an oxidized aluminum finish, can sensibly decrease the internal temperature. Additional countermeasures, to be investigated in future analyses, might include thermal tapes or louvers.

\subsection{Telecommunication system}

The radio link with Clipper can be divided into short-range mode and long-range mode. The long-range mode is used for inter-satellite distances higher than $10^{5} \mathrm{~km}$ and up to $10^{6} \mathrm{~km}$, such as those reached during the pre-insertion phase. In this situation, it might be necessary for the main spacecraft to modify its attitude to point the HGA to ETP. The short-range mode, instead, is nominally employed during the orbital phase when the relative distance is lower than $10^{5} \mathrm{~km}$ and it is used for the gravity science experiment and for TT\&C.

We carried out the link budget considering X-band for both the radio link modes because it is compliant with the communication system of the main spacecraft. Moreover, the radio frequency output power of the probe has been set to $1 \mathrm{~W}$, to be delivered by the conical horn MGAn. Including a minimum margin of $3 \mathrm{~dB}$ [37], the signal-overnoise ratio is always larger than $25 \mathrm{dBHz}$ (computed at the largest distance, $10^{6} \mathrm{~km}$ ). The complete communication system on ETP includes the 20-dBi MGAn for nominal communications and two LGAs that provide a quasi-omnidirectional antenna gain pattern. A Radio Frequency Distribution Unit provides switches to select the desired antenna output, and the transponder is used for Doppler measurements during the ISL.

Since the mass, volume and power consumption of currently available transponders for deep space exploration do not satisfy the system requirements, this component requires further specialized development. However, we selected NASA's IRIS V2 Deep Space Transponder for budgeting purposes and adapted its power consumption to the radio frequency output of the probe. This is the optimal transponder in terms of mass, power consumption, and volume, and it can be used as a guideline during the development of the final component.

\subsection{On-board data handling}

ETP's daily data production is composed of the magnetic field vector measurements (which account for $415 \mathrm{kbit} /$ day) and the subsystem telemetry, for a total of $0.5 \mathrm{Mbit} / \mathrm{day}$. Since, according to Clipper's nominal trajectory, communication windows happen once every 15 days, adequate mass storage capability is provided by 4, 16-Mbit Rad- 


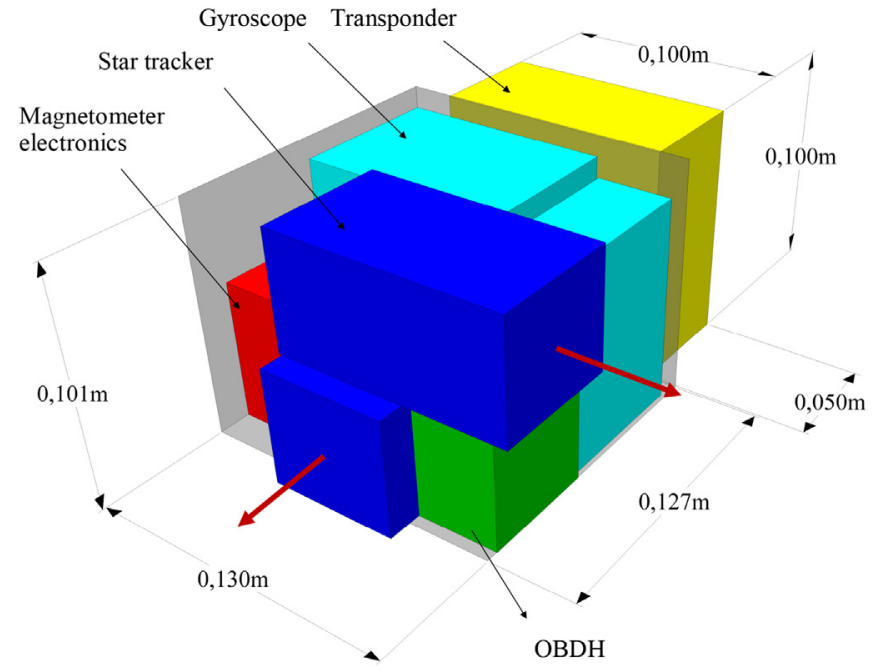

Fig. 6. Radiation vault internal configuration; the red arrows indicate the optical axes of the star trackers. (For interpretation of the references to colour in this figure legend, the reader is referred to the Web version of this article.)

Hard SRAM units. The on-board computer shall provide fault detection and correction capabilities, especially as the spacecraft does not communicate directly with the Earth. To this aim, the Spacecraft Mode Controller monitors the subsystem telemetries and switches between the different modes when programmed or when receiving a command. The software is embedded into the centralized CPU along with the power-intensive attitude determination filter. The selected CPU is a 32 - bit SPARC V8 Rad-Hard processor that largely fulfills the estimated software requirements with an 86 Million Instructions Per Second (MIPS) throughput.

\subsection{Radiation shielding}

The major sources of ionizing radiation are the electrons trapped in Jupiter's magnetic field. A radiation dose analysis showed that the Total Ionizing Dose (TID) for ETP's mission is over $4000 \mathrm{krad}$ under $0.5 \mathrm{~cm}$ of equivalent aluminum shielding. Since this value is high enough to exceed the radiation hardness of almost every space component, a radiation vault has been selected as the primary shielding method. The most sensitive components are the avionics, the attitude determination sensors, the magnetometer electronics, the transponder and the PCDU electronics.

The design of the vault has been iterated many times with particular attention to the radiation hardness of the least hardened component and the shielding thickness (and thus mass). The final configuration has then been determined after a system trade-off that included the constraints imposed by the attitude determination sensors and the transponder. In particular, the STs shall both look to the free space, while the SSs shall be placed on the Sun-facing panel. Both the STs and the SSs also require a clear field of view. Furthermore, the positioning of the transponder is influenced by possible electro-magnetic interference with the rest of the avionics. A simplified view of the radiation vault configuration is shown in Fig. 6. The geometry of the vault has been conceived in such a way to obtain the least overall dimensions with reasonable margins for the currently unknown geometry of the PCDU. Moreover, a margin of $1 \mathrm{~cm}$ has been applied to every face of a component that is adjacent with a wall.

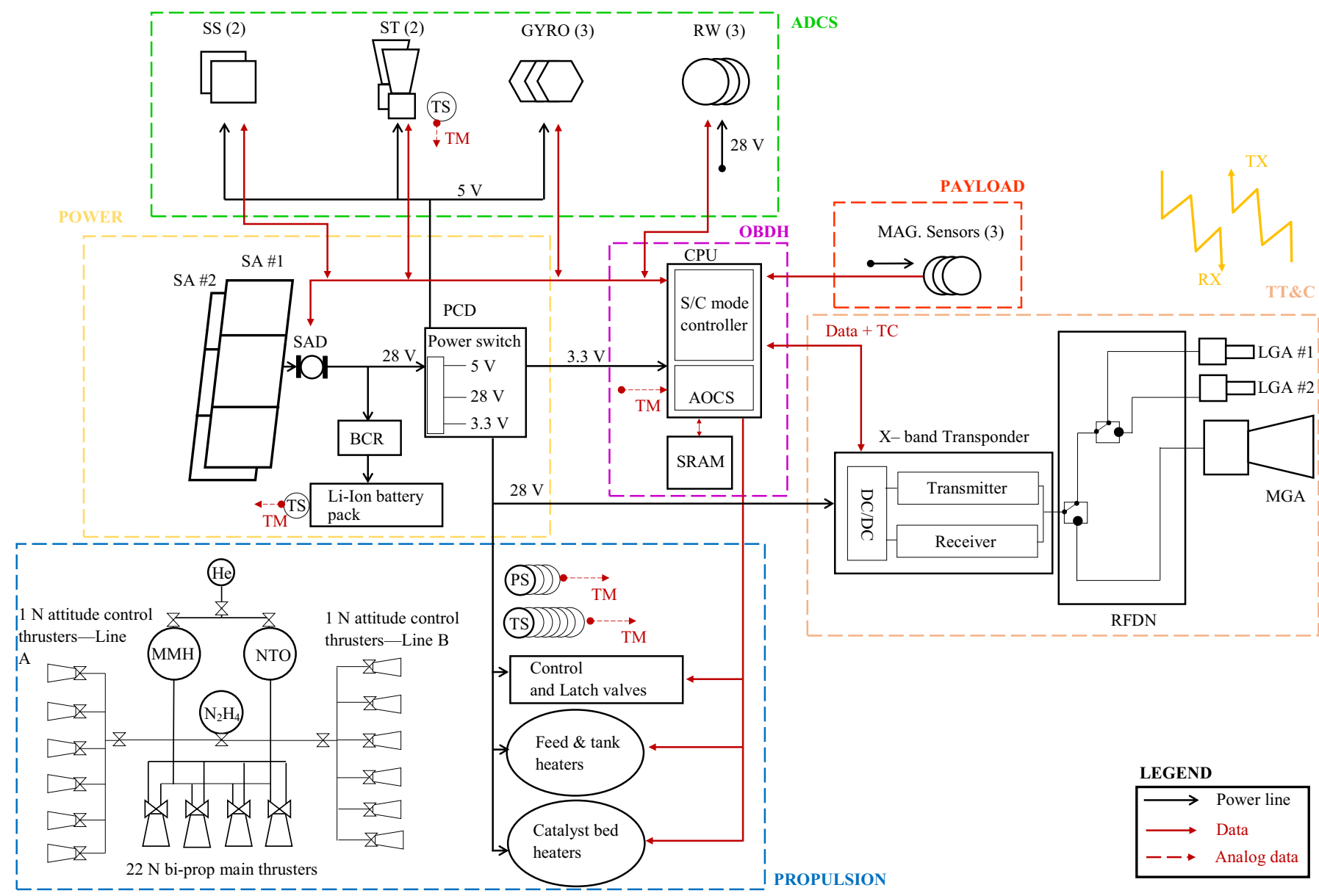

Fig. 7. ETP functional block diagram. 
Table 4

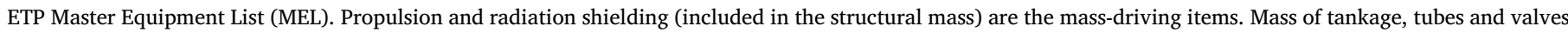

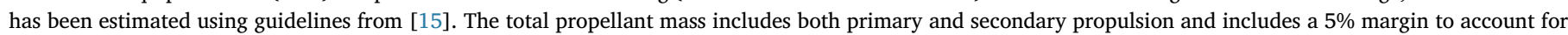
residual propellant.

\begin{tabular}{|c|c|c|c|c|c|c|c|c|}
\hline Subsystem/Component name & Quantity & Unit mass (kg) & CBE mass (kg) & MGAl (\%) & Predicted mass (kg) & Margin (\%) & Allowable mass (kg) & TRL \\
\hline Propulsion (dry) & & & & & & & 27.75 & \\
\hline Tankage and valves, lines & 1 & / & 16.01 & / & / & 30 & 20.80 & 9 \\
\hline Main engine & 4 & 0.45 & 1.80 & 5 & 1.89 & 30 & 2.46 & 9 \\
\hline RCS engine & 12 & 0.33 & 3.96 & 5 & 4.15 & 30 & 5.39 & 9 \\
\hline AOCS & & & & & & & 5.94 & \\
\hline Sun Sensor & 2 & 0.33 & 0.66 & 5 & 0.69 & 30 & 0.90 & 9 \\
\hline Star Tracker & 2 & 0.35 & 0.70 & 15 & 0.81 & 30 & 1.05 & 6 \\
\hline Gyroscope & 3 & 0.14 & 0.42 & 15 & 0.48 & 30 & 0.63 & 6 \\
\hline Reaction wheel & 3 & 0.75 & 2.25 & 15 & 2.59 & 30 & 3.36 & 6 \\
\hline EPS & & & & & & & 33.64 & \\
\hline Solar panels & 1 & 18.70 & 18.70 & 10 & 20.57 & 30 & 26.74 & 9 \\
\hline Power cells & 24 & 0.13 & 3.12 & 15 & 3.59 & 30 & 4.66 & 6 \\
\hline PCDU hardware & 1 & 1.50 & 1.50 & 15 & 1.73 & 30 & 2.24 & 6 \\
\hline TCS & & & & & & & 0.54 & \\
\hline MLI & 1 & 0.38 & 0.38 & 10 & 0.42 & 30 & 0.54 & 9 \\
\hline OBDH & & & & & & & 1.00 & \\
\hline CPU and SRAM & 1 & 0.70 & 0.70 & 10 & 0.77 & 30 & 1.00 & 9 \\
\hline Payload & & & & & & & 0.42 & \\
\hline Structure & & & & & & & 109.45 & \\
\hline Aluminum structure & 1 & 24.10 & 24.10 & 10 & 26.51 & 30 & 34.46 & / \\
\hline Radiation shielding & 1 & 50.16 & 50.16 & 15 & 57.68 & 30 & 74.99 & / \\
\hline Telecommunications & & & & & & & 6.56 & \\
\hline MGAn & 1 & 3.00 & 3.00 & 5 & 3.15 & 30 & 4.09 & 9 \\
\hline LGA & 2 & 0.30 & 0.60 & 5 & 0.63 & 30 & 0.82 & 9 \\
\hline Transponder & 1 & 1.20 & 1.20 & 10 & 1.32 & 30 & 1.72 & 6 \\
\hline Total dry mass & & & & & & & 185.35 & \\
\hline Propellant & & & 71.55 & & & 35 & 96.59 & \\
\hline Total wet mass & & & & & & & 281.94 & \\
\hline
\end{tabular}

The TID at the center of the vault is $75 \mathrm{krad}$ under $5 \mathrm{~cm}$ of aluminum, which becomes $100 \mathrm{krad}$ after imposing a 30\% TID margin. STs do not currently comply with this value, but the component manufacturer is already planning to upgrade their radiation hardness to at least the required value. The Sun Sensors have been independently shielded with a $3.5 \mathrm{~cm}$-thick aluminum enclosing because their intrinsic radiation hardness is significantly higher than the other components ( $300 \mathrm{krad}$ ), thus leading to mass savings. The TID at the center of the SSs box is $150 \mathrm{krad}$.

\section{Results and discussion}

Table 4 shows the Master Equipment List for ETP. The CBE mass for each component is listed along with the MGAl percentage, predicted mass, mass margin and final allowable mass. The table also shows the TRL of the selected components based on their current design. The total wet mass with MGAl and margin is $>40 \%$ larger than the system-level CBE, and, with the architecture described in Section 5, is well under $300 \mathrm{~kg}$. This result satisfies the constraint imposed by the maximum available added mass on Europa Clipper as per the NASA announcement, and demonstrates that a small probe with minimal payload can still sensibly increase the science return of a larger flyby spacecraft to Europa. Although we considered Europa Clipper's reference mission profile, the large margin imposed on each subsystem assures that the mass budget presented in this analysis is robust under different assumptions on the ISL frequency and duration. Therefore, regardless of whether the mass allowance on Europa Clipper is still being considered by NASA, the ETP concept and the resulting system analysis presented here and in [12] are of general significance for the demonstration of the capabilities of the inter-satellite link to augment gravity science, and can be flexibly adapted to future planetary science missions aimed at determining the interior structure of Europa. The mass margin accounts also for the intrinsic uncertainty related to the main spacecraft's configuration and the design of the mechanical interfaces with ETP.

We point out some issues related to ETP's system design. Note that ETP has been conceived as a small secondary probe aimed at augmenting the mother spacecraft: given the simplicity of its payload, a non-redundant design has been selected for each subsystem except for the propulsion system (due to the need for attitude control). However, internal redundancy at the software- and component-levels has been envisioned. Furthermore, we did not carry out a complete cost breakdown in the framework of this feasibility study as the main aim has 
been to demonstrate that the concept is technically feasible and fits in the 250-300-kg range. The total cost associated to the addition of ETP to a planetary mission to Europa would probably be driven by the technology advancement required to develop a transponder with low power consumption tailored for deep space exploration. However, thanks to the ever-increasing interest in small spacecraft for deep space applications, we expect this technology gap to be filled in the coming years. A detailed cost estimation shall be carried out if the ETP concept will be considered for further analysis, either for the Europa Clipper mission or for a future mission to the Jovian moon.

\section{Conclusions}

This paper has presented the results of a feasibility study for Europa Tomography Probe, proposed as a small Europa orbiter aimed at characterizing the moon's interior structure. The global subsurface ocean present on Europa is still a mystery for planetary scientists, as its characteristics are largely unknown. Europa Clipper will be launched by NASA in the 2020s to perform a series of flybys of Europa and study its internal and surface composition. Due to its intermittent encounters with the moon, Clipper will not be able to resolve the time-variable magnetic field (induced by Jupiter on the global ocean conductor) at the relevant frequencies ( $11 \mathrm{~h}$ and $85 \mathrm{~h}$ ), preventing an accurate determination of the ocean's depth and conductivity. Following NASA's announcement of a 250-300-kg mass allowance on Europa Clipper, we proposed ETP as a secondary probe equipped with only a magnetometer and a transponder as payloads. In a parallel paper, we show that by continuously measuring the magnetic and gravity fields of Europa, ETP would be able to determine the ocean's characteristics with high accuracy, thus increasing the scientific return of the main mission. The use of an inter-satellite link will also improve the radial positioning of the main spacecraft and will be beneficial to its altimetric measurements [12]. Here we have demonstrated that the concept is technically feasible with a total mass under $300 \mathrm{~kg}$, accounting for a conservative design margin that ensures that ETP fits within the NASA requirement. The same concept can also be suitable as an added payload to other planetary science missions dedicated to the investigation of Europa's interior structure (and other icy moons as well). In fact, regardless of whether the mass allowance on Europa Clipper is still being considered, the ETP concept presented here and in [12] is of general interest for the minimal payload suite that is yet sufficient to answer crucial scientific questions about Europa's habitability. Furthermore, ETP would be a major example of the use of small probes for deep-space exploration, in that it would enable scientific discoveries otherwise inaccessible with larger flyby spacecraft.

\section{Acknowledgements}

This work has been carried out under partial sponsorship from Agenzia Spaziale Italiana (ASI). We would like to thank the personnel of the Radio Science Lab at Sapienza University of Rome, for many helpful comments and support. A particular acknowledgement goes to Alberto Anselmi and his group at Thales Alenia Space Italy (Turin) for contributing to this work with crucial advices.

\section{References}

[1] K.K. Khurana, et al., Induced magnetic fields as evidence for subsurface oceans in Europa and Callisto, Nature 395 (1998) 777-780.
[2] M.H. Carr, et al., Evidence for a subsurface ocean at Europa, Nature 391 (1998) $363-365$.

[3] J.D. Anderson, et al., Europa's differentiated internal structure: evidence from four Galileo encounters, Science 281 (5385) (1998) 2019-2022.

[4] C.F. Chyba, C.B. Phillips, Europa as an abode of life, Orig. Life Evol. Biosph. 32 (2002) 47-68.

[5] National Research Council, Vision and Voyages for Planetary Science in the Decade 2013-2022, The National Academies Press, Washington, D. C., 2011.

[6] C. Zimmer, K.K. Khurana, M.G. Kivelson, Subsurface oceans on Europa and Callisto: constraints from Galileo magnetometer observations, Icarus 47 (2000) 329-347.

[7] J.M. Wahr, et al., Tides on Europa, and the thickness of Europa's icy shell, J. Geophys. Res. 111 (2006) 2156-2202 E12005.

[8] Europa Study Team, Europa Study 2012 Report: Europa Orbiter Mission, Tech, rep. JPL, California, 2012.

[9] ESTEC CDF Team, CLEO/P: Assessment of a Jovian Moon Flyby Mission as Part of NASA's Clipper Mission, Tech. Rep. ESTEC, The Netherlands, 2015.

[10] B. Buffington, Trajectory design for the Europa clipper mission concept, AIAA/AAS Astrodynamics Specialist Conference, San Diego, CA, 2014 4-7 August.

[11] T. Bayer, et al., Europa Clipper Mission: the habitability of an icy moon, IEEE Aerospace Conference, Big Sky, MT, 2015 7-14 March.

[12] M. Di Benedetto, et al., Augmenting NASA Europa clipper by a small probe: Europa tomography probe (ETP) mission concept, submitted to, Acta Astronaut. 165 (2019), https://doi.org/10.1016/j.actaastro.2019.07.027.

[13] L. Roth, et al., Transient water vapor at Europa's south Pole, Science 343 (6167) (2014) 171-174.

[14] Asmar, et al., Small spacecraft for planetary atmospheric, surface and interior structure using radio links, IEEE Aerospace Conference Proceedings (2018), 3-10 March 2018, Big Sky (MT), 2018.

[15] J.R. Wertz, W.J. Larson, Space Mission Analysis and Design, third ed., Microcosm Press, 1999.

[16] P. Fortescue, J. Stark, S. Graham, Spacecraft System Engineering, third ed., J. Wiley \& Sons, 2003.

[17] M.R. Patel, Spacecraft Power Systems, CRC Press, 2005.

[18] G.P. Sutton, O. Biblarz, Rocket Propulsion Elements, seventh ed., J. Wiley \& Sons, 2000.

[19] C.D. Brown, Spacecraft Propulsion, AIAA, 1996.

[20] M.J. Sidi, Spacecraft Dynamics and Control, Cambridge University Press, 2000.

[21] Mission Design Division Staff, Small Spacecraft Technology State of the Art, Tech. Rep. Ames Research Center, CA, 2014.

[22] JPL-D-17868 (REV.1), JPL Guideline: Design, Verification/Validation and Operations Principles for Flight Systems, (16 February 2001).

[23] ANSI/AIAA S-120A-2015, Standard: Mass Properties Control for Space Systems, (21 January 2016).

[24] S.R. Hirshorn, Expanded Guidance for NASA Systems Engineering. Volume 1: Systems Engineering Practices, Tech. rep National Aeronautics and Space Administration, 2016.

[25] K.K. Khurana, et al., Searching for liquid water in Europa by using surface observatories, Astrobiology 2 (1) (2002) 93-103.

[26] M.K. Dougherty, Personal Communication, (2016) 3.

[27] S.W. Asmar, et al., Ultra-stable oscillators for planetary entry probes, Proc. Int. Workshop 'Planetary Probe Atmospheric Entry and Descent Trajectory Analysis and Science', Lisbon Portugal, 2003.

[28] S.W. Asmar, et al., Spacecraft Doppler tracking: noise budget and accuracy achievable in precision radio science observations, Radio Sci. 40 (2005) RS2001.

[29] A. Eremenko, et al., Europa clipper spacecraft configuration evolution, IEEE Aerospace Conference, Big Sky, MT, 2014.

[30] L. Federici, et al., Preliminary capture trajectory design for Europa Tomography probe, Int. J. Aerosp. Eng. (2018) 2018.

[31] A. Boutonnet and G. Varga, reportJuice - Jupiter icy Moons Explorer Consolidated Report on Mission Analysis (CReMA), JUI-ESOC-MOC-RP-001, Issue 3, Revision 2, 25/04/2017.

[32] J.P.S. Carvalho, et al., Low-altitude, near-polar and near-circular orbits around Europa, Adv. Space Res. 49 (2012) 994-1006.

[33] D.J. Scheeres, et al., Stability analysis of planetary satellite orbiters: application to the Europa orbiter, J. Guid. Control Dyn. 24 (4) (2001) 778-786.

[34] M. Cinelli, et al., Polynomial equations for science orbits around Europa, Celest. Mech. Dyn. Astron. 122 (2015) 199-212.

[35] National Research Council, Assessment of Planetary Protection Requirements for Spacecraft Missions to Icy Solar System Bodies, The National Academies Press, Washington, D. C., 2012.

[36] Carsten Baur, Status Report on the Solar Cells Characterization under Low Intensity Low Temperature Conditions, Tech. Rep. ESTEC, Noordwijk, The Netherlands, 2014.

[37] ECSS-E-50-05A, Space Engineering, Radio Frequency and Modulation, Tech. Rep, ESTEC, Netherlands, 2003. 